\title{
Listeria y listeriosis: un desafío de los nuevos tiempos
}

\author{
SERGIO ALCAYAGA(1) y BÁRBARA HOTT ${ }^{(2)}$
}

\section{IMPORTANCIA EN SALUD PÚBLICA}

Como consecuencia de los cambios en el sistema de vida y en los hábitos alimentarios, las enfermedades causadas por el consumo de alimentos contaminados han surgido como una causa importante de morbimortalidad a nivel mundial. Se han descrito alrededor de 250 agentes causales de enfermedades transmitidas por alimentos (ETA), entre los que se incluyen bacterias, virus, hongos, parásitos, priones, toxinas y metales. En USA se estiman en 76 millones los casos anuales de ETA, que significan 325.000 hospitalizaciones y 5.000 muertes, lo cual representa costos significativos dentro de los gastos en salud ${ }^{1}$.

La listeriosis es considerada como una enfermedad infecciosa de origen alimentario con una tasa de mortalidad de 30\%. Estudios muestran que el origen de casos esporádicos y epidémicos de listeriosis en humanos es principalmente de origen alimentario ${ }^{2}$.

Aun cuando en 1953 se reconoció la participación de un alimento en la transmisión de la enfermedad ${ }^{3}$, no fue sino hasta 1983 que se comunicó el primer brote de listeriosis relacionado a alimentos ${ }^{4}$.

A fines de los años setenta y a comienzos de los años ochenta, el número de aislamientos de listeria spp. en el mundo comenzó a incrementarse y desde 1983 en adelante, una serie de brotes epidémicos en humanos en Norteamérica y Europa, claramente establecieron a la listeriosis como una importante enfermedad de transmisión alimentaria. Los alimentos más frecuentemente implicados son quesos suaves y productos lácteos, patés y embutidos, pescado ahumado, ensaladas, "delicatessen" y, en general, alimentos producidos industrialmente, productos refrigerados listos para consumo que se comen sin cocinar o recalentar. Esto indica que listeria spp. es una seria amenaza a la seguridad de los alimentos y la sitúa entre las categorías de microorganismos de mayor preocupación de la industria alimentaria ${ }^{5}$. En la actualidad se estima que Listeria monocytogenes es la principal causa de muerte originada por bacterias de origen alimentario en USA. En ese país se registran 2.500 casos aproximadamente de listeriosis humana por año, incluyendo 500 muertes $^{6}$.

Es posible que algunas tecnologías habitualmente empleadas en la industria alimentaria como la refrigeración, deshidratación, congelación y descongelación, tratamiento con sal, $\mathrm{pH}$ ácido, exposición a desinfectantes y otras sustancias antimicrobianas, etc., influyan en el estado fisiológico y en la virulencia de este patógeno, seleccionando subpoblaciones bacterianas resistentes al estrés y más virulentas?

Observaciones indican que la virulencia de L. monocytogenes varía de un aislamiento a otro, como lo muestra el caso de un brote de listeriosis en el que estaba implicada una cepa presente en el alimento con un bajo número de bacterias, además se encontró que una segunda cepa no asociada con el brote y también presente se hallaba en números más altos, por lo que se sugiere que probablemente la virulencia de estas dos cepas fuese diferente ${ }^{8}$. Sin embargo, hasta ahora, todas las cepas de L. monocytogenes son consideradas potencialmente patógenas.

(1) Secretaría Regional Ministerial de Salud. Región Metropolitana. Moneda 1025. 5 Piso. Santiago. Chile. ssmsepi@hotmail.com

(2) Secretaría Regional Ministerial de Salud. Región Metropolitana. Santiago. Chile. 
Las posibles explicaciones de la emergencia de la listeriosis humana transmitida por alimentos, como un asunto de máxima importancia en Salud Pública, comprende variados temas, entre ellos los cambios importantes en la producción, procesamiento y distribución de alimentos, la utilización cada vez mayor de la refrigeración como medio de conservación primaria de ellos, cambios en los hábitos de alimentación de la población, particularmente con respecto a la comodidad de tenerlos ya preparados y de un incremento del número de personas consideradas de alto riesgo de sufrir la enfermedad (ancianos, gestantes, recién nacidos, inmunodeprimidos) 9 .

\section{MODOS DE PRESENTACIÓN CLÍNICA DE LA ENFERMEDAD}

En la listeriosis se describen dos tipos de cuadros: el gastroentérico y el invasivo. El cuadro gastroentérico puede tener una presentación que puede oscilar desde portadores sin síntomas (2-5\%), hasta individuos con signos gastrointestinales moderados a severos. En el cuadro invasivo ${ }^{10}$ los signos y síntomas en el inicio pueden incluir fiebre, calambres abdominales, diarrea, fatiga, dolor de cabeza y dolor muscular ${ }^{11}$. Se presentan diversas formas clínicas de listeriosis, tales como: listeriosis de la mujer durante el embarazo (muchas veces causa de aborto); listeriosis del recién nacido o granulomatosis infantiséptica, meningoencefalitis y romboencefalitis por listeria que se presenta en neonatos y adultos mayores de 50 años; listeriosis cutánea; listeriosis septicémica con faringitis y mononucleosis; listeriosis óculo glandular; listeriosis cervicoglandular, y otras formas de listeriosis que se describen como infecciones focales que provocan artritis, osteomielitis, abscesos espinales o cerebrales, peritonitis, colecistitis y endocarditis ${ }^{12}$.

\section{EPIDEMIOLOGÍA}

El género Listeria se encuentra mundialmente distribuido. Está representado por siete especies, pero sólo dos son de interés en la patología humana y animal: L. monocytogenes y L. ivanovii. La especie más importante tanto para el hombre como los animales es la L. monocytogenes ampliamente difundida en el medio ambiente. Ha sido aislada de diferentes sitios ambientales, como: suelo, plantas, barro, pasto, aguas servidas, aguas de arroyos, heces humanas y animales, además de una gran variedad de alimentos. Hay casos indiscutibles de transmisión directa de la infección de los animales al hombre, como se registra en partos de animales infectados que fueron asistidos por individuos sin guantes ${ }^{13}$.

La epidemiología de la listeriosis no es completamente conocida. La mayoría de los investigadores la considera una enfermedad común al hombre y a los animales y no como una zoonosis propiamente tal. Es probable que los animales contribuyan al conjunto de listerias en la naturaleza y sobre todo a su distribución. Se describen períodos de incubación desde 11 a 70 días, con una mediana de 31 días $^{14}$.

Una característica importante de esta bacteria es su capacidad de desarrollarse a temperaturas bajas; con un $\mathrm{pH}$ entre 6 y 9 puede multiplicarse a temperaturas de entre $3^{\circ} \mathrm{C}$. y $45^{\circ} \mathrm{C}$. Sin embargo, aun cuando es una bacteria ubicua y con gran resistencia a condiciones extremas, la enfermedad es poco frecuente en la población general, afectando principalmente a personas en edades extremas de la vida, embarazadas y pacientes inmunodeprimidos. Se sabe que la dosis infecciosa de L. monocytogenes es de, al menos, $10^{2}$ bacterias viables en el caso de los grupos de riesgo y que esta cifra aumenta hasta $10^{4}$ en el caso de población sana. Sin embargo, aún existen muchas lagunas en la comprensión del proceso dosis respuesta de la listeriosis humana y del papel que juega la virulencia de la cepa implicada, así como su interacción con el hospedador ${ }^{15}$. 
Tabla 1. Algunos brotes de listeriosis en el mundo

\begin{tabular}{|c|c|c|c|c|c|c|c|}
\hline LUGAR & AÑO & $\begin{array}{c}\mathrm{N}^{\circ} \\
\text { CASOS }\end{array}$ & $\begin{array}{c}\mathrm{N}^{\circ} \\
\text { MUERTES }\end{array}$ & $\begin{array}{c}\text { LETALIDAD } \\
\%\end{array}$ & SEROTIPO & ALIMENTO & REFERENCIA \\
\hline $\begin{array}{l}\text { Halifax, } \\
\text { Canadá }\end{array}$ & 1981 & 41 & 18 & 48 & $4 b$ & Coles & Schlech et al. 1983 \\
\hline Massachussets & 1983 & 49 & 14 & 29 & $4 \mathrm{~b}$ & Leche & Fleming et al. 1985 \\
\hline Vaud, Suiza & $\begin{array}{l}1983- \\
1987 \\
\end{array}$ & 122 & 34 & 28 & $4 b$ & Queso & Bula et al. 1995 \\
\hline California, & 1985 & 142 & 48 & 24 & $4 b$ & Queso & Linnan et al. 1995 \\
\hline Reino Unido & $\begin{array}{l}1989- \\
1990\end{array}$ & 300 & 0 & 0 & $4 b$ & Paté & $\begin{array}{l}\text { McLauchlin et al. } \\
1991\end{array}$ \\
\hline Francia & 1992 & 279 & 88 & 32 & $4 b$ & $\begin{array}{l}\text { Lengua de } \\
\text { cerdo }\end{array}$ & Jacquet et al.1995 \\
\hline Francia & 1993 & 39 & 0 & 0 & $4 b$ & $\begin{array}{l}\text { Paté } \\
\text { de cerdo }\end{array}$ & Goulet et al. 1995 \\
\hline Francia & 1995 & 36 & 0 & 0 & $4 b$ & Queso blando & Goulet et al. 1995 \\
\hline $\begin{array}{l}\text { Multiestados } \\
\text { USA }\end{array}$ & $\begin{array}{l}1998- \\
1999 \\
\end{array}$ & 40 & 4 & 10 & $4 b$ & Carne cortada & CDC 1998 \\
\hline Finlandia & $\begin{array}{l}1988- \\
1999 \\
\end{array}$ & 25 & 6 & 24 & $3^{\mathrm{a}}$ & Manteca & Lyytikainen et al. 2000 \\
\hline Francia & 1999 & 29 & 7 & 24 & NI & $\begin{array}{l}\text { Lengua de } \\
\text { cerdo }\end{array}$ & WHO 2000 \\
\hline $\begin{array}{l}\text { Multiestados } \\
\text { USA }\end{array}$ & 2000 & 29 & 4 & 14 & $4 b$ & Pavo cortado & CDC 2002 \\
\hline $\begin{array}{l}\text { Carolina } \\
\text { del Norte }\end{array}$ & $\begin{array}{l}2000- \\
2001\end{array}$ & 12 & 0 & 0 & $4 b$ & Queso & CDC 2002a \\
\hline $\begin{array}{l}\text { Multiestados } \\
\text { USA }\end{array}$ & 2002 & 46 & 7 & 15 & NI & Pollo y pavo & CDC 2002a \\
\hline $\begin{array}{l}\text { Quebec, } \\
\text { Canadá }\end{array}$ & 2002 & 17 & 0 & 0 & NI & Queso & Gaulin et al. 2003 \\
\hline Canadá & 2008 & 56 & 20 & 36 & $\mathrm{NI}$ & Carne & $\begin{array}{l}\text { Public Health Agency } \\
\text { of Canada }\end{array}$ \\
\hline
\end{tabular}

NI: Serovariedad no informada.

\section{LISTERIOSIS EN CHILE}

Chile sigue la tendencia mundial con respecto a la ocurrencia de casos relacionados al consumo de alimentos. La modalidad de vigilancia epidemiológica de esta patología es de laboratorio a través del Instituto de Salud Pública (Decreto $\mathrm{N}^{\circ}$ 158), no siendo obligatoria la notificación de casos clínicos. El Reglamento Sanitario de los Alimentos no incluye este patógeno entre las especificaciones de vigilancia para productos de consumo nacional. Sólo se vigila en preparados cárnicos listos para el consumo y productos lácteos de exportación, esta situación hace difícil obtener datos epidemiológicos para establecer tendencias. 
Se han realizado en Chile algunos estudios en alimentos. En la Tabla 2 se exponen los resultados de uno de ellos.

Tabla 2. Listeria monocytogenes aislada de alimentos. Santiago. Chile. $1990-1997^{16}$

\begin{tabular}{|l|c|c|}
\hline ALIMENTO & $\begin{array}{c}\mathbf{N}^{\circ} \\
\text { MUESTRAS }\end{array}$ & $\begin{array}{c}\mathbf{N}^{\circ} \\
\text { MUESTRAS } \\
\text { POSITIVAS }\end{array}$ \\
\hline Helados & 603 & 21 \\
\hline Quesos blandos & 256 & 2 \\
\hline Quesos duros & 155 & 0 \\
\hline Biberones $^{\circ}$ & 229 & 0 \\
\hline Cecinas $^{\mathrm{a}}$ & 634 & 23 \\
\hline Mariscos & 268 & 31 \\
\hline Total & 2145 & 77 \\
\hline
\end{tabular}

a: Incluidos 443 embutidos (20 contaminados), 160 patés (2 contaminados) y 31 jamones (1contaminado)

En la segunda etapa de este estudio (2000) se analizaron 709 muestras de ensaladas en venta en supermercados de Santiago. Fueron positivas a L. monocytogenes $26 \%$ de las muestras de ensaladas congeladas y $10,5 \%$ en ensaladas frescas de supermercado. El ISP, el año 2008 en el marco de investigación de un brote analizó 262 muestras de variados alimentos, obteniendo resultados positivos para listeria en: ensalada de rúcula, ensalada de tomate lechuga, jamón serrano, choclo congelado, arvejas congeladas, salmón ahumado y quesos blandos.

Según estadísticas chilenas publicadas en la Revista Chilena de Obstetricia y Ginecología, entre 1982 y 1987 la incidencia de la enfermedad fue de 0,42 por 1.000 recién nacidos vivos, con una letalidad de 17,6\%. Dado que esta enfermedad no es una patología de declaración obligatoria, modalidad universal o centinela, según se explicó anteriormente, no se dispone de antecedentes de su situación epidemiológica en el país, en años recientes.

Se informó por parte de profesionales de la Clínica de la Pontificia Universidad Católica de Chile, la existencia de cuatro casos en embarazadas, infectadas por L. monocytogenes, de un total de 16 casos ocurridos en ese centro asistencial entre los años 2001 y $2005^{17}$.

En junio del año 2008, un equipo de médicos infectólogos de la Clínica Alemana de Santiago, envió a la Seremi de Salud de la Región Metropolitana una alerta en atención a un aumento de casos de listeriosis diagnosticados en ese establecimiento asistencial.

Con el propósito de investigar la situación referida, la Seremi de Salud de la RM realizó una investigación epidemiológica de los aislamientos de L. monocytogenes, que se pidió informar para el periodo 2006-2008 a los principales establecimientos de salud de la Región Metropolitana. Este proceso comenzó en el mes de junio de 2008 como una búsqueda retroactiva de casos y posteriormente continúo con la notificación y registro de casos nuevos.

El Ministerio de Salud en su página web tiene disponible el informe de la situación de la listeriosis en Chile, el que es permanentemente actualizado. Los datos que se presentan a continuación en relación al brote de la Región Metropolitana son en su mayoría extractos de estos informes y comunicaciones personales del equipo investigador del brote.

Se notificaron al 31 de diciembre de 2008, 119 casos de infección por L. monocytogenes (cifra cinco veces superior a la de años anteriores), siendo provenientes de establecimientos públicos (34\% de los casos) y privados (66\% de los casos). 
Figura 1. Aislamientos de L. monocytogenes 2006-2008 en la R.M.

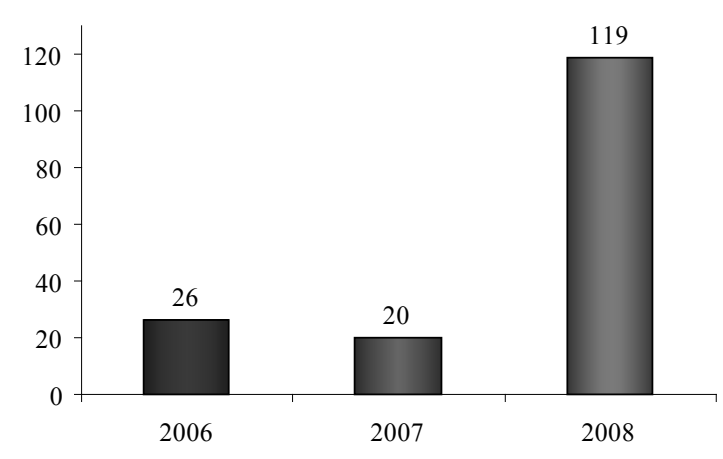

Fte. Subdepto. Epidemiología SEREMI R.M.

El Instituto de Salud Publica realizó un estudio utilizando la técnica de electroforesis de campo pulsado (PFGE) para la tipificación genética de las cepas de listeriosis provenientes de muestras clínicas informadas el año 2008. La utilidad de este tipo de análisis, en el estudio de un brote en el que se desconoce su origen, se basa - fundamentalmente - en la posibilidad de caracterizar los casos ocasionados por un mismo patógeno según su tipo genético, lo que permite diferenciar aquellos que posiblemente pudiesen tener un origen común y de esta manera optimizar en tiempo y recursos. Además, se ha demostrado que el riesgo de contraer listeriosis no sólo depende del inóculo, sino también del subtipo molecular de la cepa implicada ${ }^{18}$.

El ISP determinó que el $55 \%$ de los aislamientos de la Región Metropolitana el año 2008 correspondían a una cepa genéticamente relacionada (clon 009), considerada la cepa epidémica causante del brote.

La investigación epidemiológica y de laboratorio permitió concluir que el alimento causante del brote correspondía a quesos de las variedades Brie y Camembert de la marca Chevrita. El Instituto de Salud Pública analizó 82 muestras de estos quesos, obtenidos de la planta de producción, resultando ser los únicos alimentos con el mismo patrón genético 009 encontrado en las cepas clínicas.
La mayoría de los casos reportados de esta enfermedad, a nivel mundial, se debe al consumo de leche no pasteurizada o subproductos elaborados con la misma. Brotes ocurridos a pesar de la pasteurización dejan claro que el proceso no elimina el riesgo de contaminación posterior $\mathrm{y}$, por lo tanto, los alimentos pasteurizados conllevan el mismo peligro que la leche cruda ${ }^{19}$.

La caracterización de los casos relacionados al brote de la RM, muestra que el 44\% corresponde a embarazadas, $17 \%$ a adultos mayores, $17 \%$ recién nacidos, $8 \%$ inmunosuprimidos, $8 \%$ adultos mayores con patologías de base y $6 \%$ personas sin factor de riesgo aparente.

Figura 2. Sistema de salud de establecimientos donde consultaron casos 2008. RM.

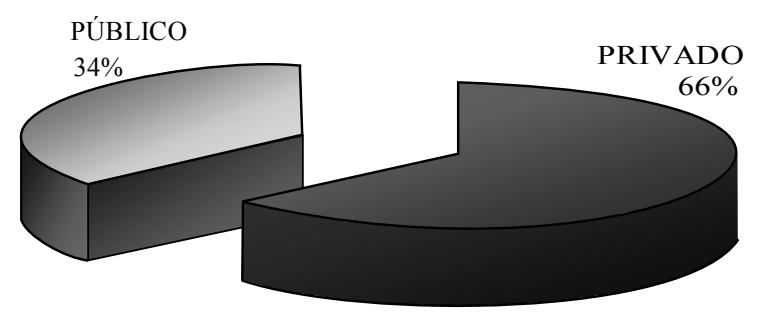

Fte. Subdepto. Epidemiología SEREMI R.M.

\section{CONCLUSIONES}

Actualmente se están produciendo cambios en los patrones epidemiológicos de diversas enfermedades infecciosas, incluidas las enfermedades transmitidas por alimentos. Estos cambios pueden tener múltiples causas, algunas de las cuales se explican por el cambio climático y los cambios de estilos de vida asociados al aumento de la jornada laboral, lo que se traduce en preferir alimentos listos para el consumo o aquellos que son preparados en casinos o restaurantes. 
La listeriosis es una enfermedad que genera casos aislados y brotes epidémicos, siendo más frecuente los casos esporádicos y aislados. Se describe como una patología de los países desarrollados y de aquellos en vías de desarrollo. Esto debido a que generalmente la mayoría de los brotes están asociados a alimentos sometidos a refrigeración y a procesos industrializados. La globalización y el intercambio de productos entre los países han generado una variada oferta de alimentos importados que lentamente han comenzado a formar parte de los hábitos alimenticios de los chilenos. Conjuntamente las empresas nacionales han visto esta situación como una oportunidad para producir y ofrecer estos mismos productos a precios más atractivos.

Aquel paradigma que por muchos años ha existido no sólo en las personas relacionadas al área de salud, sino en la comunidad en general, en relación a asociar las enfermedades transmitidas por alimentos a la pobreza y la falta de tecnologías para la elaboración y conservación de alimentos, influye en el poco conocimiento que existe por parte de comunidad de las enfermedades transmitidas por alimentos relacionadas a otras causas. Es evidente la necesidad de crear conciencia con respecto a la asociación de otros factores en la emergencia y re-emergencia de las enfermedades del este nuevo siglo.

El brote ocurrido el año 2008 en la Región Metropolitana, es el brote más grande descrito en Chile causado por L. monocytogenes asociado a alimentos. En este caso el alimento involucrado fueron quesos producidos industrialmente, que no eran de consumo masivo por lo que el brote afectó, principalmente, a un segmento específico de la población, lo que refuerza la asociación establecida entre la emergencia de enfermedades infecciosas poco frecuentes con los cambios culturales.

Estos cambios obligan a reformular periódicamente las actividades de promoción de salud y de control de las enfermedades, así como los reglamentos, normativas y medidas sanitarias que rigen la producción y expendio de alimentos. Estas medidas deben ir actualizándose al ritmo de los cambios en los estilos de vida de la población. En este contexto, se reproducirán las recomendaciones que respecto de listeriosis son entregadas en su página web por los Centros de Control de Enfermedades (CDC) de los Estados Unidos.

(http://www.cdc.gov/nczved/dfbmd/disease_ listing/listeriosis_gi.html\#reducerisk)

1) Recomendaciones para población general:

- Cocine perfectamente los alimentos de origen animal, como vacuno, cerdo o ave.

- Lave minuciosamente los vegetales crudos antes de su consumo.

- Mantenga carnes crudas separadas de los vegetales, de los alimentos cocidos y de los alimentos listos para el consumo.

- Evite la leche no pasteurizada, así como alimentos producidos con leche no pasteurizada.

- Lave sus manos, cuchillos y tablas de cortar después de manipular alimentos crudos.

- Consuma lo más rápidamente posible los alimentos perecibles y aquellos que se expenden listos para el consumo.

2) Recomendaciones para las personas con riesgo elevado. Tales como embarazadas y personas con su sistema inmune debilitado (deben agregarse a las recomendaciones enumeradas previamente):

- No coma hot dogs (vienesas), fiambres o embutidos, a menos que se recalienten hasta temperatura de cocción.

- Evite que el líquido proveniente de los paquetes de alimentos crudos (vienesas, carnes) entre en contacto con otros alimentos, utensilios, y superficies donde se preparan alimentos.

- Lávese las manos después de manipular vienesas, carnes crudas, fiambres y embutidos.

- No coma los quesos blandos tales como queso Feta, Brie y Camembert, quesos con vetas azules, o quesos del tipo mexicano tales 
como Blanco, Fresco y Panela, a menos que tengan etiquetas que indiquen claramente que se hacen con leche pasteurizada.

- No coma los patés o pastas de carne refrigeradas. Pueden ser consumidos los patés y las pastas de carnes en conserva.

- No coma los mariscos y pescados ahumados refrigerados, a menos que estén contenidos en un plato cocinado, como un caldillo. Los productos del mar ahumados refrigerados, tales como salmones, trucha, pescado blanco, bacalao, atún o caballa, se etiquetan a menudo como "Nova-style," "lox," "kippered," "smoked," o "jerky." Pueden ser consumidos los productos del mar ahumados en conserva.

\section{REFERENCIAS}

1. MEAD P, SLUTSKER L, DIETZ V, MCCAIG LF, BRESEE JS, SHAPIRO C ET AL. 1999 Food-related illness and death in the United States. Emerg Infect Dis. 5: 607 - 625.

2. BOERLIN P., F. BOERLIN-PETZOLD, E. BANNERMAN, J. BILLE, T. JEMMI. 1997. Typing Listeria Monocytogenes Isolates from Fish Product and Human Listeriosis Cases. Applied and Environmental Microbiology. 4: 1338 - 1343.

3. WHO Microbiological Risk Assessment Series, No. 5. Risk assessment of Listeria monocytogenes in ready-to-eat foods: technical report.. Rome 2004.

4. SCHLECH W. F. $3^{\text {rd }}$, Lavigne P M, Bortolussi R A, Alien A C, Haldane E V, Wort AJ, et al. 1983. Epidemic listeriosis-evidence for transmission by food. N Engl J Med. 308: 203-206.

5. VÁSQUEZ- BOLAZ J., M. KHUN, P. BERCHE, T. CHAKRABORTY, G. DOMÍNGUEZ-BERNAL, B. GONZÁLEZ-ZORN, J. WELAND, J. KREFT. 2001. Listeria Pathogenesis and Molecular Virulence Determinants. Clinical Microbiology Reviews. 14: 564- 584.

6. MEAD, P.S., E. F. DUNNE, L. GRAVES, M. WIEDMAN, M. PATRICK, S. HUNTER, E. SALEHI., F. MOSTSHARI, A. CRAIG, P. MSHAR, T. BANNERMAN, B. D. SAUD-
ERS, P. HAYES, W. DEWITT, P. SPARLING, P. GRIFFIN, D. MORSE, L. SLUTSKER, AND B. Swaminathan. 2006. Nationwide outbreak of listeriosis due to contaminated meat. Epidemiol. Infect. 134: 744 - 751.

7. KATHARIOU S. 2002. Listeria monocytogenes virulence and pathogenicity, a food safety perspective. J Food Prot 65: 1811-29.

8. CHICO-CALERO I, SUÁREZ M, GONZÁLEZZORN B, SCORTTI M, SLAGHUIS J, GOEBEL W. 2002. a bacterial homolog of the microsomal glucose-6-phosphate translocase, mediates rapid intracellular proliferation in Listeria. Proc Natl Acad Sci USA 99: 421-6.

9. SWAMINATHAN B. 2001. Listeria monocytogenes. Food microbiology: Fundamentals and Frontiers, Second Edition, Doyle M.P., Beuchat L.R. \& Montville T.J., eds. ASM Press, Washington, DC, USA., 383 - 409.

10. F.A.O. MARTÍNEZ I., D. JAMES, H. LORÉAL. 2004. Application of modern analytical techniques to unsure seafood safety and authenticity. Distributed as and advanced copy of FAO fisheries technical paper.

11. TAGE A. 1999. Listeriosis: Recognizing it, treating it, preventing it. Cleveland Clinic Journal of medicine. 66: 375-380.

12. GAESCHE E., E. SOTO. 1991. Evaluación de un método de aislamiento de Listeria monocytogenes de pescado contaminado in Vitro. Acta microbiológica 3: 43-48.

13. ACHA P., B SZYFRES. 2001. Publicación Científica $\mathrm{N}^{\circ}$ 580. Zoonosis y enfermedades transmisibles comunes al hombre y a los animales. Tercera Edición. O.P.S/O.M.S. Washington, D.C, U.S.A., 186 - 196.

14. SCHUCHAT A., B. SWAMINATHAN, C.V. BROOME. 1991. Epidemiology of human listeriosis. clin microbiol. Rev. 4: 169 - 183.

15. ILSI Research Foundation, Risk Scince Institute. 2005 Achieving continuos improvement in reductions in foodborne listeriosis-a risk-based approach. J Food Prot 68:1932-94.

16. CORDANO A.M, J. ROCOURT 2001. Ocurrance of Listeria monocytogenes in Food in Chile. International Jornal of Food Microbiology 70:175-178.

17. LARRAÍN D, ABARZÚA F, DE JOURDAN F, MERINO P, BELMAR C, GARCÍA P. 2008. 
Infecciones por Listeria monocytogenes en mujeres embarazadas: Experiencia del Hospital Clínico de la Pontificia Universidad Católica de Chile. Rev Chil Infect; 25: 337-42.

18. CHEN Y, ROSS WH, GRAY MJ, WIEDMANN M, WIEDMANN, WHITING RC, SCOTT VN. 2006. Attributing risk to Listeria monocytogenes subgroups: dose response in relation to genetic lineages. J Food Prot 69: 335-44.
19. LUNDÉN J, TOLVANEN R, KORKEALA H. 2004. Human listeriosis outbreaks linked to dairy products in Europe. J Dairy Sci 87: E6-E12.

Recepción: 24 de septiembre de 2008

Aprobación: 21 de enero de 2009

Usted puede comentar éste y otros artículos publicados en la Revista Chilena de Salud Pública, enviando un correo electrónico a revistasp@med.uchile.cl 